\title{
Segmentation of Corneal Endothelial Cells Contour by Means of a Genetic Algorithm
}

\author{
Fabio Scarpa ${ }^{1}$ and Alfredo Ruggeri ${ }^{1}$ \\ ${ }^{1}$ Department of Information Engineering, University of Padova, Padova, Italy \\ \{fabio.scarpa, alfredo.ruggeri\} eunipd.it
}

\begin{abstract}
Corneal images acquired by in-vivo microscopy provide clinical information on the cornea endothelium health state. The reliable estimation of the clinical morphometric parameters requires the accurate detection of cell contours in a large number of cells. Thus for the practical application of this analysis in clinical settings an automated method is needed. We propose the automatic segmentation of corneal endothelial cells contour through an innovative technique based on a genetic algorithm, which combines information about the typical regularity of endothelial cells shape with the pixels intensity of the actual image. Ground truth values for the clinical parameters were obtained from manually drawn cell contours. Results show that an accurate automatic estimation is achieved: for each parameter, the mean difference between its manual estimation and the automated one is always less than $4 \%$, and the maximum difference is always less than $7 \%$.
\end{abstract}

Keywords: Corneal images, image segmentation, confocal microscopy

\section{$1 \quad$ Introduction}

The normal human corneal endothelium is a single layer of uniformly sized cells with a predominantly hexagonal shape, but this regular tessellation is affected by age and pathologies [1]. Thus, the analysis of the main morphometric parameters of corneal endothelium provides clinical information capable to describe the cornea health state. Namely, endothelial cell density (ECD), polymegethism (differences in cell size expressed as fractional standard deviation of cell areas), and pleomorphism or hexagonality coefficient (fraction of hexagonal cells over the total number of cells) are commonly used as parameters to quantitatively characterize the endothelial cells' condition.

In order to make this analysis practical in clinical settings, a computerized method that fully automates the segmentation procedure would be needed. The fundamental problem with automated endothelial analysis is to correctly identify the cells' contour, a necessary prerequisite for the estimation of the clinical parameters [2]. The automatic recognition of the cell boundaries is a challenging task because of the noise that make the contour difficult to be distinguished even by an expert, and the substantial differences between an image and the other as regards size and appearance of the

X. Chen, M. K. Garvin, J. Liu, E. Trucco, Y. Xu (Eds.): OMIA 2015, Held in Conjunction with MICCAI 2015, Munich, Germany, Iowa Research Online, pp. 25-32, 2015. Available from: http://ir.uiowa.edu/omia/2015_Proceedings/2015/ 
cells, due to different state of health of the cornea. Whereas ECD can generally be estimated with acceptable accuracy, the quantitative estimation of pleomorphism and polymegethism is significantly affected by errors in contour detection even in few cells, making the reliable estimation of these parameters quite difficult [3].

Several computer programs have been proposed to accomplish this task [4], even if to the best of our knowledge the software used in clinical practice are only semiautomated, or work in a non-clinical context, e.g., with stained cells [5]. In the former case, the cell border detection provided by the computer needs to be revised by the user to correct inaccuracies by manual adjustment. It has been reported that at least 75 cells per image should be evaluated for a reliable estimation of clinical parameters [6]. Although manual correction improves the accuracy of this estimation, it is tedious and time-consuming and therefore usually impractical in a clinical setting. This often leads the user to reduce the number of outlined cells to just a few tens, greatly affecting the accuracy of estimated parameters and thus the reliability of the clinical outcome.

We propose here a reliable, fully automated algorithm for the segmentation of the endothelial cell contours.

\section{$2 \quad$ Material}

Fifteen images of corneal endothelium were acquired with a specular endothelial microscope (SP-3000P, Top-con Co., Japan) from both healthy and pathological subjects. These images differ from each other by noise (i.e. differences in illumination drift artifact and unfocused areas), cells size (i.e. normal, medium and high ECD) and cells shape and area (i.e. normal, medium and high pleomorphism and polymegethism). The images cover an area of $0.25 \times 0.5 \mathrm{~mm}$ and were saved as 8bits, 240 x 480 pixels grayscale images (Fig. 1).

In order to assess the accuracy of the morphometric parameters estimated by the computerized procedure, ground truth reference values were obtained by estimating the parameters on manually segmented images. For each image, all visible cell contours were manually traced with care by using a public-domain image manipulation program (GIMP v. 2.8, http://www.gimp.org), so as to outline the polygonal shape of each cell. In each image, the segmented cells contour cover an area of about $0.1 \mathrm{~mm}^{2}$, enough to allow a reliable estimation of the morphometric parameters. 

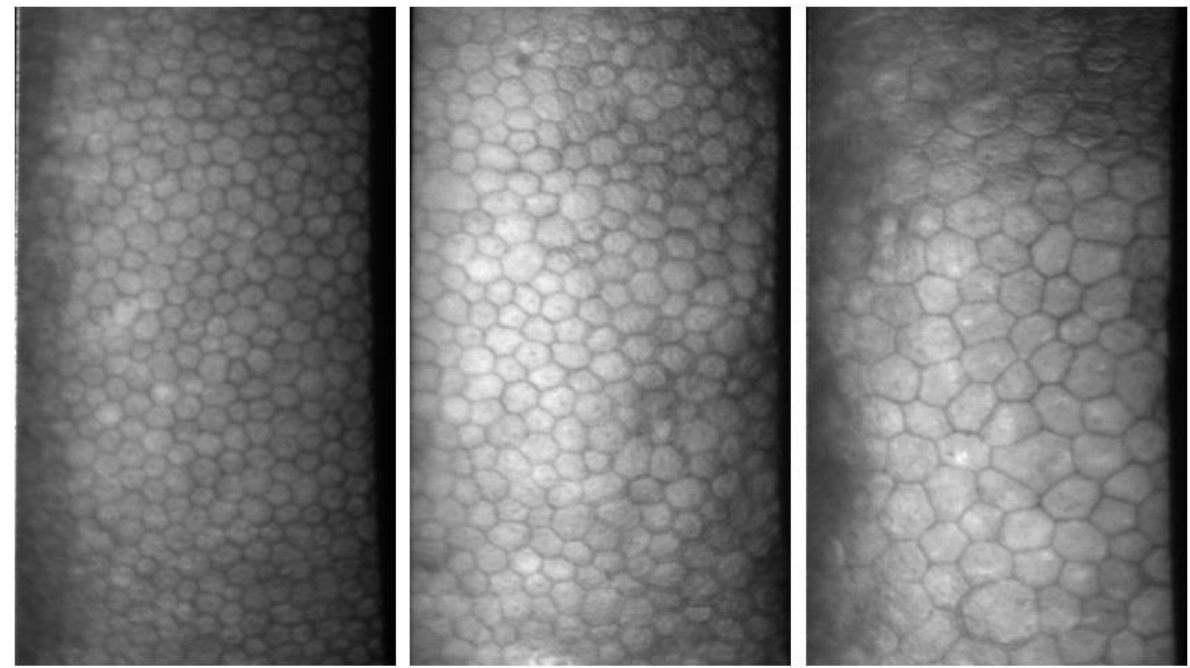

Fig. 1. Representative example of corneal endothelial images acquired by a normal subject (left), a subject with high polymegethism (middle) and a subject with high ECD (right).

\section{Methods}

Cells appear in the image as relatively regular polygons with different sizes, orientations and numbers of sides. Pixel intensity in the contour (dark) is different from the intensity in the inner body (light) of the cell. The rationale of the proposed approach is to combine information about regularity and pixel intensity, thus using a-prior knowledge and a-posteriori knowledge. Indeed, regularity can be considered as apriori information, since it is a well-known feature of endothelial cells (guaranteed in each image, albeit with different grades), while pixel intensity is an a-posteriori information, since it is relative to the specific region of the specific image under investigation.

\subsection{Pre-processing}

In order to improve the quality of corneal images, each pixel in the image is convolved with three customized 2-dimensional kernels at different scale and orientations. Each of the kernels has been specifically designed to provide a high response when its position, scale and orientation match those of the corresponding structure: vertex, side, or body of the cell [7]. Thus, three images were derived from the original image under investigation, in which vertexes, inner bodies and sides of the cells are respectively highlighted (Fig 2). 

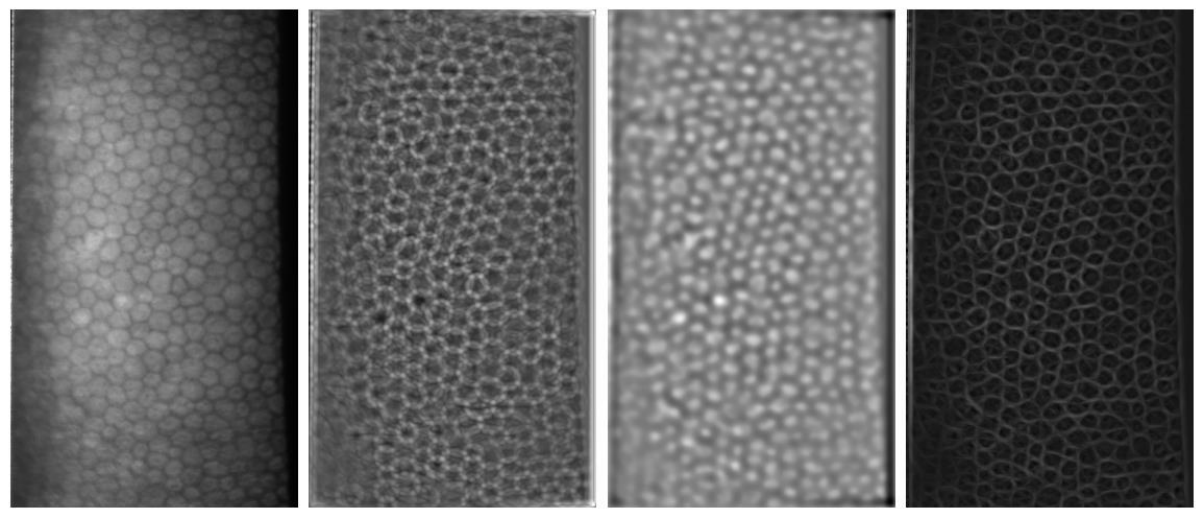

Fig. 2. From left to right: original image; image with highlighted vertexes; image with highlighted cell bodies; image with highlighted cell sides.

\subsection{Contour segmentation by a Genetic Algorithm}

Cells contour are detected using a genetic algorithm. It is a method for solving optimization problems based on a selection process that mimics biological evolution. It randomly modifies individuals from the current population to produce the children for the next generation. Over successive generations the population evolves toward an optimal solution. For the specific application, a small set of vertexes (i.e. individuals) forming regular hexagons is used as starting population. At each step, the location of each vertex is randomly modified. The reliabilities of each old vertex (parent) and the corresponding modified vertex (children) are evaluated and compared by considering both the correspondence with the actual image and the regularity of the polygons. In particular, the evaluation of each vertex is based on a cost function, whose first term (relative to the a-posteriori knowledge) is composed by indexes computed as the mean pixel intensity corresponding to the location of:

- the vertex in the image with highlighted vertexes;

- the vertex in the image with highlighted bodies;

- the sides connected with the vertex in the image with highlighted sides.

Pixel intensity is evaluated on all three filtered images, so that any errors (i.e. a not highlighted vertex, body or side) in an image have little influence on the overall result. The second term of the cost function is relative to the a-priori knowledge about regularity. The indexes identified as the most descriptive of the regularity of each vertex are:

- the ratio between the lengths of its sides;

- the ratio between the angles formed by its sides;

- the ratio between distances of the vertex from the center of the polygons to which it belongs; 
- the ratio between the mean distance of the actual vertex from the center of the polygons to which it belongs and the mean distance of all vertexes from their respective polygons.

The first three indexes take into account local features, relative only to a specific vertex, while the last index is a global feature, since it takes into account the entire structure. Furthermore, each polygon is evaluated and modified, by adding new vertexes, or by splitting, merging, or deleting some existing vertexes or modifying some connection between them: the evaluation is again based on the cost function of the old and the modified vertexes, choosing the ones that provide the best cost, i.e. combination between regularity and pixel intensity. Thus, the initial regular hexagons evolve into polygons with possibly different number and positions of vertexes. An example of the evolution of the vertexes can be seen in Fig. 3. The number of polygons is stepwise increased and the algorithm stops when the whole area of the image is covered with polygons. The final entire population of vertexes forms a set of polygons that fit the underlying cells contours. The final contour segmentation of representative images can be seen in Fig. 4.

\subsection{Post processing}

A final post-processing step is performed to exclude the polygons (and consequently vertexes) whose vertexes have a lower score with respect to the mean score of all vertexes in the image. This step allows to avoid the cell contour detection in unfocused areas or in proximity of illumination artifacts, in which a reasonable contour detection is not possible.

The proposed algorithm has been implemented using the Matlab language. The run time of the actual prototype version is in the order of tens of minutes per image. The run time can be reduced by means of parallel computing (not implemented yet) or by porting the algorithm to a more performing programming language (e.g. $\mathrm{C}++$ ).
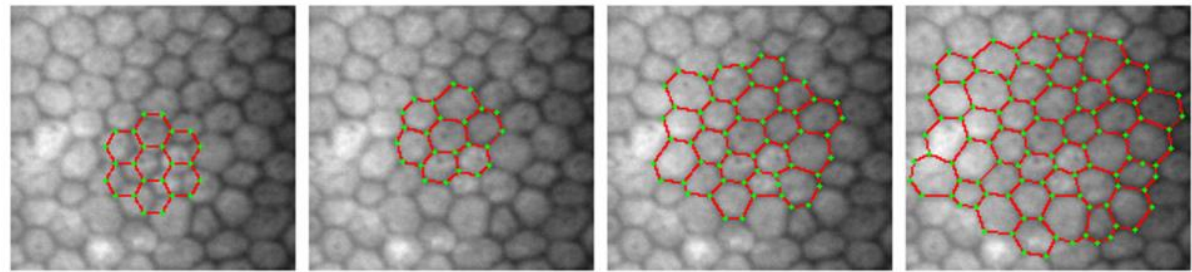

Fig. 3. Representative example of the cell segmentation's evolution:. From left to right: starting regular hexagons; change in the position of each vertex considering both its correspondence with the actual image and the regularity of the polygons; increased number of polygons and subsequent amendment of the vertexes. 

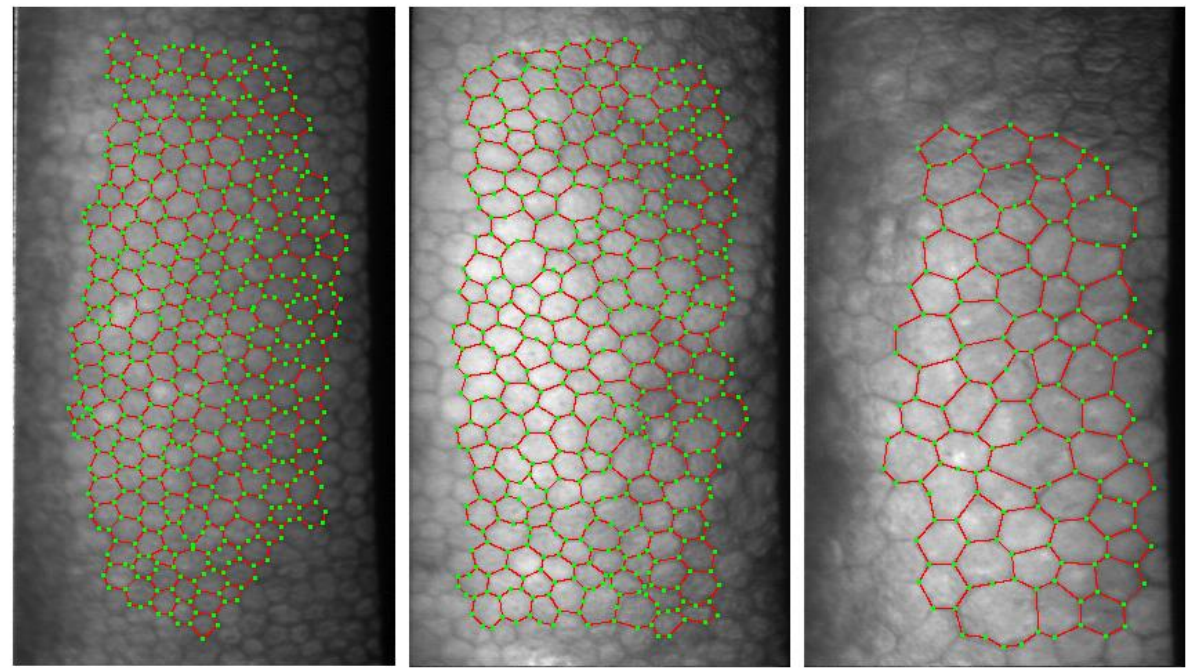

Fig. 4. Final segmentation of corneal endothelial cells contour in three representative images.

\section{$4 \quad$ Results}

For each pair of segmented images (automated and manual), only contours positioned in the same region were considered, in order to ensure that differences in the estimation of the clinical parameters were due to differences in the segmentation process and not to differences in the selected cells. From these contours, the estimation of ECD, pleomorphism, and polymegethism was carried out:

- ECD was computed as the sum of individual cell areas divided by the total number of cells;

- Pleomorphism was computed by counting for each cell the number of neighboring cells (cells along the border of the ROI were excluded from this computation) and taking the percentage of cells with hexagonal shape;

- Polymegethism was computed as the standard deviation of all cell areas divided by the mean of all cell areas.

Table 1 reports the statistics (mean, standard deviation, minimum and maximum) of the differences in morphometric parameters as estimated from the automatic segmentation and from the manual one. These results show that the proposed algorithm provides an accurate automatic estimation of the clinical parameters, whose mean difference from the manual estimation is always less than $4 \%$ and the maximum difference is always less than $7 \%$. Moreover, they allow an improvement in the estimation of the clinical parameters, especially pleomorphism and polymegethism, compared to that obtained by other techniques applied on similar corneal endothelial images, where differences between the estimated manual and automatic parameters were often greater than $7 \%$ for pleomorphism and $10 \%$ for polymegethism $[5,7]$. 
Table 1. Summary results for ECD, pleomorphism, and polymegethism estimations. Differences between automatic and manual assessment of the parameters are expressed as difference (diff), its percent (diff \%), absolute difference (abs diff) and its percent (abs diff \%). ECD values are in cells $/ \mathrm{mm}^{2}$, pleomorphism and polymegethism are percent values.

\begin{tabular}{rrrrr}
\hline ECD & diff & diff $\%$ & abs diff & abs diff $\%$ \\
\hline Mean & 6,13 & $0,45 \%$ & 17,73 & $0,82 \%$ \\
Sd & 23,72 & $1,29 \%$ & 16,31 & $1,08 \%$ \\
Min & $-32,00$ & $-0,96 \%$ & 0,00 & $0,00 \%$ \\
Max & 66,00 & $4,38 \%$ & 66,00 & $4,38 \%$ \\
\hline Pleomorphism & diff & diff $\%$ & abs diff & abs diff $\%$ \\
\hline Mean & $-0,31$ & $-0,74 \%$ & 1,83 & $3,13 \%$ \\
Sd & 2,29 & $3,88 \%$ & 1,33 & $2,27 \%$ \\
Min & $-3,60$ & $-5,89 \%$ & 0,00 & $0,00 \%$ \\
Max & 3,80 & $6,24 \%$ & 3,80 & $6,24 \%$ \\
\hline Polymegethism & diff & diff $\%$ & abs diff & abs diff \% \\
\hline Mean & 1,25 & $3,33 \%$ & 1,45 & $3,95 \%$ \\
Sd & 1,10 & $2,94 \%$ & 0,79 & $1,93 \%$ \\
Min & $-1,50$ & $-4,70 \%$ & 0,00 & $0,00 \%$ \\
max & 2,80 & $6,86 \%$ & 2,80 & $6,86 \%$ \\
\hline & & & &
\end{tabular}

\section{Conclusion}

We presented here a system for the estimation of cornea endothelium morphometric parameters that requires no user intervention. The proposed totally automatic algorithm appears capable of reliably obtaining the cell contour in regions containing hundreds of small cells (normal subject) as well as few great cells (subject with low ECD) or cells with varying size (subject with high polymegethism). The estimates of the clinical parameters provided by the proposed algorithm are in very good agreement with ground truth, obtained with a careful manual analysis.

As regards ECD, its estimation can actually be performed with acceptable accuracy by many computerized systems, as the presence of some errors in cell detection is of limited impact on the final ECD value. On the contrary, as already noted by several authors, e.g. [3], the quantitative estimation of pleomorphism and polymegethism is significantly affected by errors in contour detection even in few cells, making the reliable estimation of these parameters quite difficult. A possible solution is the manual correction of cell borders, but this involves a significant amount of work, e.g., on about 50 to 75 per cent of cell borders [3]. The capability of the proposed algorithm to correctly segment cell contours allows to correctly estimate also pleomorphism and polymegethism.

Even if an exhaustive evaluation on a greater number of images and on images acquired by subjects with different pathologies are needed, these preliminary results demonstrate the ability of the proposed genetic algorithm to adapt to the different shapes and sizes of cells, as well as to the different features and quality of the images. 
For the reasons explained above, in large experimental studies with hundreds of images, the experimenter usually restricts him/herself to measure only ECD. The system we propose can be extremely valuable in these studies, as it allows to reliably estimate also the other two morphometric parameters. Moreover, it can process images containing hundreds (and not tens) of cells and thus provide a much higher accuracy for the estimated parameters. This could be further increased by performing the analysis on several images per subject, each positioned at slightly different locations in central cornea, and then averaging the results.

\section{References}

1. L. Mastropasqua, M. Nubile, "Confocal Microscopy of the Cornea," Ed. SLACK Incorporated, 2002, pp. 1-16.

2. L. Imre and A. Nagymihaly, "Reliability and reproducibility of corneal endothelial image analysis by in vivo confocal microscopy," Graefe's Archive for Clinical and Experimental Ophthalmology, 2001.

3. M.J. Doughty and B.M. Aakre, "Further analysis of assessments of the coefficient of variation of corneal endothelial cell areas from specular microscopic images," Clinical and Experimental Optometry, 2008.

4. D.V. Patel and C.N. McGhee, "Quantitative analysis of in vivo confocal microscopy images: A review," Survey of Ophthalmology, 2013.

5. A. Ruggeri, F. Scarpa, M. De Luca, C. Meltendorf, J. Schroeter, "A system for the automatic estimation of morphometric parameters of corneal endothelium in alizarine redstained images," British Journal of Ophthalmology, 2010.

6. M.J. Doughty, A. Muller and M.L. Zaman, "Assessment of the reliability of human corneal endothelial cell-density estimates using a noncontact specular microscope," Cornea, 2000.

7. E. Poletti E and A. Ruggeri, "Segmentation of Corneal Endothelial Cells Contour through Classification of Individual Component Signatures,” IFMBE Proceedings, 2014. 